\title{
Modification of View Field in Wireless Multimedia Sensor Networks
}

\author{
Jingjing Xiao \\ School of Electrical, Electronic \\ and Computer Engineering, \\ University of Birmingham, UK
}

\author{
Hongyu Ren \\ Centre for Precision Technology \\ University of Huddersfield, UK
}

\author{
Wusheng Luo \\ College of Mechatronics \\ Engineering and Automation, \\ National University of Defense \\ Technology, China
}

\begin{abstract}
Numerous micro-devices are interconnected in Wireless Multimedia Sensor Networks (WMSNs), such as surveillance. While due to view field of these multimedia sensors is oriented, blind spots caused by an occlusion is unavoidable, which will impact on surveillance service quality. For reducing this affection and figuring out a real sensing area, detailed formulas are given in this paper to compute real points. Four steps are designed to detect salient area, which act as potential obstacles. After that, an algorithm utilizing rectangle to approximate the detected areas is proposed, which consider hemline as an intersecting line between earth and objects. Then using this intersecting line as a benchmark, a maximum prism container is employed to find out the blind area. Experiments show that even for monocular image, this algorithm can efficiently find out the real view field of nodes. In addition, by considering physical obstacles in wireless multimedia sensors, this paper contributes to $3 \mathrm{D}$ field of view study.
\end{abstract}

\section{Keywords}

Multimedia sensor nodes, Maximum prism container, Field of view

\section{INTRODUCTION}

The availability of low-cost, small-scale imaging sensors indicate the development of Wireless Multimedia Sensor Networks (WMSNs), which allows retrieving video streams and enhances existing sensor network applications in both civilian and military areas [1]. Demand of these multimedia sensors is driven by the necessity of providing comprehensive information pertaining to a specific region of interest [2]. However, these multimedia sensors produce deluge of data, requiring more extensive processing capacity and higher transmission rates, which puts more challenges on both hardwires and algorithms in WMSNs [3]. Different from ordinary sensors, multimedia sensors are direction-sensitive with a line of sight (LOS) [4]. This means that their sensing areas are based on many factors such as location, orientation, and physical obstacles. Therefore, the conventional coverage model and wakeup mechanism for collaboration in WSN is no longer useful in WMSNs. Moreover, obstacles will cause occlusion problem. Therefore, modifying the perception model and establishing new collaborative strategy to enhance the quality of surveillance service has become an essential task in WMSNs.

This paper places its primary focus on modifying the perception model. For the installation of parameters, 3D sensing model with monocular camera is ideally conducted.
Then, these obstacles are recognized and computed by saliency detection. During this procedure, four general steps are designed. First, a rectangle is used to approximate the salient area in an image. Then, its hemline is considered as the intersecting line between ground and objects. Based on this line, a maximum prism container is utilized to find out the occlusion area. The main contribution in this paper is the modification of real 3D perception model in monocular multimedia nodes, which will be the foundation for future research about collaborative strategy. Furthermore, this paper is the first one to consider physical obstacles in 3D field of view study in WMSNs.

The remainder of this paper is structured as follows. Section 2 introduces some related work. Section 3 computes the world coordinates from image coordinates in terms of nodes mechanical structure. Considering the physical obstacles, the modification of nodes' view field is provided in section 4 . In section 5, performances of this novel model are evaluated. And section 6 gives a conclusion for this work and provides future research lines.

\section{RELATED WORK}

One of the works that showed the implementation of WMSNs for a specific application is described in [5], where video cameras are networked together to fulfill the task of collecting interesting oceanographic events. For network coverage problem of multimedia sensors, Ma [6] proposes the concept of direction sensor network. It uses camera's field of view to describe sensing range, which is the maximum visible volume from cameras [7]. However, some simplifications adopted in that work are far from reality since it does not consider the obstacles. Tezcan [2] proposed a distributed algorithm to determine a node's multimedia coverage and to find the sensor orientation, which minimizes the negative effect of occlusions and overlapping regions in the sensing field. This algorithm assumes that each multimedia node knows its location information and the location of all the obstacles around it in advance, which cannot be achieved because WMSNs are usually used in unknown environment. In addition, even though there have already being some researches to focuse on the problem of coverage, all these works are mainly based on the $2 \mathrm{D}$ perception model.

In fact, both sensor nodes' perceive model and corresponded perception of the scene are 3D structures. Traditional simplified two-dimensional (2D) perception model and corresponded sensor deploy schemes can be rarely applied to physical environment directly [9]. A 3D directional sensor coverage-control model with tunable orientations is proposed in [10]. It uses virtual potential-field based coverage- 
enhancing scheme to improve the coverage performance. A multi-objective evolutionary approach for solving multiobjective 3D deployment problems in differentiated wireless sensor networks is also proposed in [11]. In [8], a spatial correlation model is presented for visual information in WMSNs, which derive a spatial correlation function and employ an entropy-based framework to describe the correlation characteristics of visual information observed by cameras with overlapped field of views. However, all above schemes mainly focus on coverage enhancement but not the model itself. Xiao [12] considers the 3D perception model for the wireless multimedia sensor network. It also proposes a coverage-enhancing algorithm to improve the efficiency of coverage, which adjusts nodes' sensing direction by tilt-angle and deviate-angle optimization. Although that work is based on 3D perception model, the model is still idealistic as it does not consider the blind areas caused by physical obstacles in their models. Without considering occlusion problem, quality of surveillance service in WMSNs is heavily limited. The previous coverage-enhancing schemes are then no longer efficient.

Therefore, this paper is focusing on the perception model of multimedia nodes.

\section{IDEAL SENSING REGION}

In order to enhance the quality of surveillance, a real sensing area should be figured out. Therefore, the scheme and character of WMSNs in this work is stated as follows:

- There is only one camera in every multimedia node, and the perception model is obtained from single static image. In other words, this work is to try to recover $3 \mathrm{D}$ information from 2D images.

- The multimedia nodes are distributed in wide unknown area, and the scenarios captured by cameras vary from one to the other, which means directly detecting the obstacles may be not possible by scenario training.

In computer vision, sensing process is usually described by pinhole camera model [13]. There is a similarity relationship between the object and projection depicted in Figure1. The physical size of objects can be obtained through multiplying the projection size in the image by a coefficient.

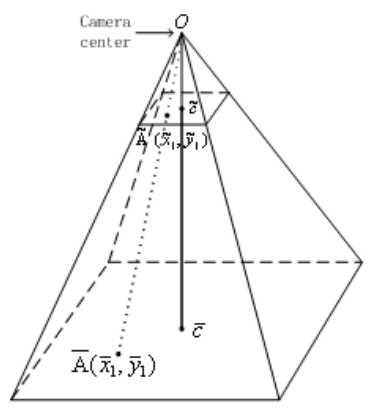

(a) Pinhole model

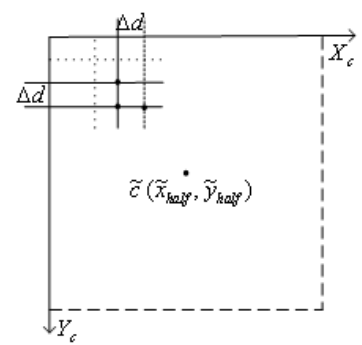

(b) Image coordinates
Figure1 Camera projection model

After calibration, natural parameters are as follows: $\Delta d$ represents the distance between neighbor pixels; the focal length of the camera $(f)$, and its height of $\left(h_{\text {ideal }}\right)$.

Via image processing, location of $\tilde{\mathrm{A}}\left(\tilde{x}_{1}, \tilde{y}_{1}\right)$ in image plane can be computed. The coordinates $\overline{\mathrm{A}}\left(\bar{x}_{1}, \bar{y}_{1}\right)$ in world plane are computed by similarity relation (1).

$$
\left[\begin{array}{l}
\bar{x}_{1} \\
\bar{y}_{1}
\end{array}\right]=\frac{\Delta d \cdot h_{\text {ideal }}}{f}\left[\begin{array}{l}
\tilde{x}_{1} \\
\tilde{y}_{1}
\end{array}\right]
$$

In [12], researchers install the camera in multimedia node as shown in Figure2 (a). In addition, another perception model whose upper perception frontier does not interact with the earth is shown in Figure2(c).

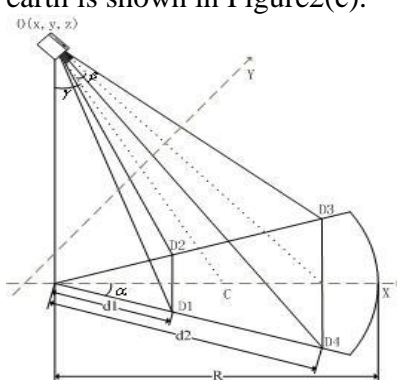

(a) Installing model I

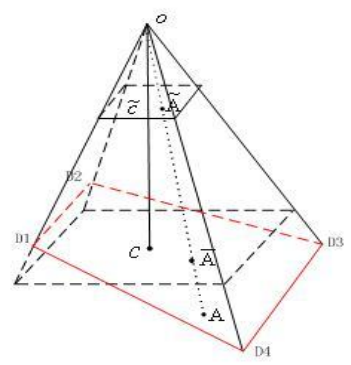

(b) Abstracted model I

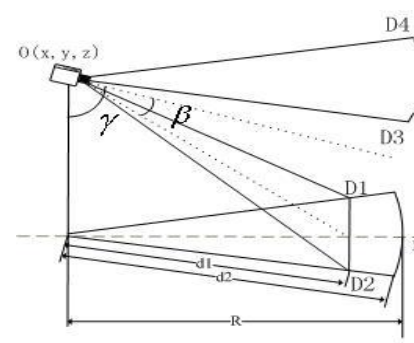

(c) Installing model II

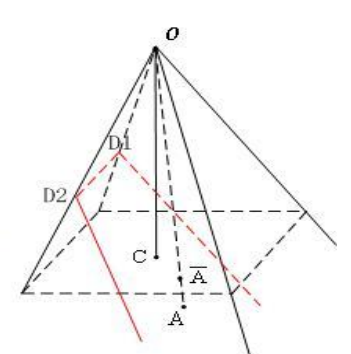

(d) Abstracted model II Figure2 Ideal perception model

In general, field of view of these two models is determined by four parameters $(\mathrm{O}, \overrightarrow{\mathrm{C}}, 2 \beta, R)$, where $\mathrm{O}=(x, y, z)$ is the location of the camera; $\vec{C}=(\gamma, \theta)$ is the sensing orientation of the multimedia sensor nodes, with the tilt angle $\gamma$ to $\mathrm{Z}$-axis from $[0, \pi / 2]$ and $\theta$ is deviation angle which characters the offset of $\vec{C}$ in $\mathrm{X}$-axis from $[0,2 \pi] ; 2 \beta$ is the maximum angle of view field in both horizontal and vertical respect; $\mathrm{R}$ is the efficient sensing radius.

For the sake of simplifying this model, the deviation angle is assumed to be zero $\theta=0$ when installing the camera. Based in this assumption, the camera height $z=h$ in $\mathrm{O}=(x, y, z)$ and tilt angle $\gamma$ can be known. Location parameters $(x, y)$ by using many sophisticated methods $[14,15]$ can as well be obtained, which are not the emphases of this paper. Parameters $2 \beta$ and $R$ are determined when cameras are manufactured.

An inclined plane is respectively utilized to intercept the model of Figure1 (a) and (c) to get what is shown in Figure2 (b) and (d), where $\bar{A}\left(\bar{x}_{1}, \bar{y}_{1}\right)$ is the projection of $A\left(x_{1}, y_{1}\right)$ in visual plane. Then, the length of $O C$ can be obtained in terms of camera height $h$ and tilt angle $\gamma$

$$
O C=h / \cos \gamma
$$

The visual plane helps reconstruct ideal sensing model, and this model can be used as medium for computing real world coordinates, which means visual point $\bar{A}\left(\bar{x}_{1}, \bar{y}_{1}\right)$ becomes a medium to compute the world point $A\left(x_{1}, y_{1}\right)$ location from point $\tilde{A}\left(\tilde{x}_{1}, \tilde{y}_{1}\right)$ in image plane.

From formula (1), $\bar{A}$ C can be computed: 


$$
\overline{\mathrm{A}} \mathrm{C}=\sqrt{\left(\tilde{x}_{1}-\tilde{x}_{\text {half }}\right)^{2}+\left(\tilde{y}_{1}-\tilde{y}_{\text {half }}\right)^{2}} \cdot \Delta d \cdot O C / f
$$

Due to this space relation, world coordinates of those points can be employed for next processing. Different locations of a point have various geometric relations, and in next section formulas based on two different situations will be deduced: points in the upper plane and the nether plane.

\subsection{Upper half plane}

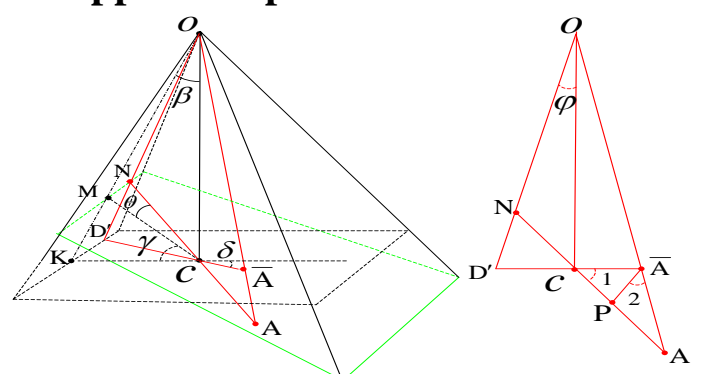

(a) Overall model (b) Abstract geometric relation Figure3 Points in the upper plane

In this model, $\mathrm{K}$ is the middle point of the edge of visual plane. Assuming the maximum angle is $\beta, O C$ being visual height, $\mathrm{CK}$ is the length, and then $\mathrm{OK}$ can be computed:

$$
\begin{aligned}
& C K=O C \cdot \tan \beta \\
& O K=O C / \cos \beta
\end{aligned}
$$

On the other hand, $\mathrm{M}$ is the corresponding point of $\mathrm{K}$ in real plane and it is in the middle of the intersection line. From triangle $O K C$, the length of $C M$ can be found using the formula (6):

$$
C M=\frac{O C}{\sin \gamma+\cos \gamma \cdot \cot \beta}
$$

The length of $\mathrm{K} M$ can be deduced via formula (7):

$$
\begin{gathered}
\mathrm{K} M=\frac{C \mathrm{M} \cdot \sin \gamma}{\cos \beta} \\
\delta=\arctan \left|\frac{\tilde{x}_{1}-\tilde{x}_{\text {half }}}{\tilde{y}_{1}-\tilde{y}_{\text {half }}}\right|
\end{gathered}
$$

Where $\delta$ is the intersection corner of the line $C \mathrm{~K}$ and $\overline{\mathrm{A}} \mathrm{D}^{\prime}$ which are both in the visual plane. After calculating $\delta$, it is quite easy to get $C \mathrm{D}^{\prime}$ :

$$
C \mathrm{D}^{\prime}=\mathrm{CK} / \cos \delta
$$

Based on right angle theorem, the length of $\mathrm{OD}^{\prime}$ is:

$$
O \mathrm{D}^{\prime}=\sqrt{\mathrm{CD}^{\prime 2}+\mathrm{OC}^{2}}
$$

Benefiting from the relation $O \mathrm{KD}^{\prime}, O \mathrm{~N}$ is denoted as:

$$
\mathrm{ON}=\frac{\mathrm{OK}-\mathrm{KM}}{\mathrm{OK}} \cdot \mathrm{OD}^{\prime}
$$

In the Figure3.b, $\varphi$ is the intersection corner of the line $O C$ and $O \mathrm{D}^{\prime}$ :

$$
\varphi=\arctan \frac{C D^{\prime}}{O C}
$$

Based on the law of cosines, NC can be computed by formula (13):

$$
\mathrm{NC}=\sqrt{\mathrm{ON}^{2}+\mathrm{OC}^{2}-2 \mathrm{ON} \cdot \mathrm{OC} \cdot \cos \varphi}
$$

In terms of the Figure3.b, the corner $\angle 1$ is:

$$
\angle 1=\arccos \frac{C D^{\prime 2}+N C^{2}-N D^{\prime 2}}{2 C D^{\prime} \cdot N C}
$$

The $\angle 2$ is denoted by formula (15):

$$
\angle 2=90^{\circ}+\angle 1-\arctan \frac{O C}{\bar{A} C}
$$

Then, ACbecome easy to compute:

$$
A C=\bar{A} C(\cos \angle 1+\sin \angle 1 \cdot \tan \angle 2)
$$

In Figure3.a, $\theta$ is the intersection corner of line $A C$ and $\mathrm{MC}$ :

$$
\theta=\arccos \left(\frac{C M}{N C}\right)
$$

Then, the world coordinates of $A$ is:

$$
A\left(x_{1}, y_{1}\right)=(\mathrm{CA} \sin \theta, \mathrm{CA} \cos \theta)
$$

\subsection{Nether half plane}

When the projection point $A$ is on the nether half plane, the geometric relation has changed (see Figure4). Therefore, previous formulas cannot be applied for computing world coordinates of $A$.

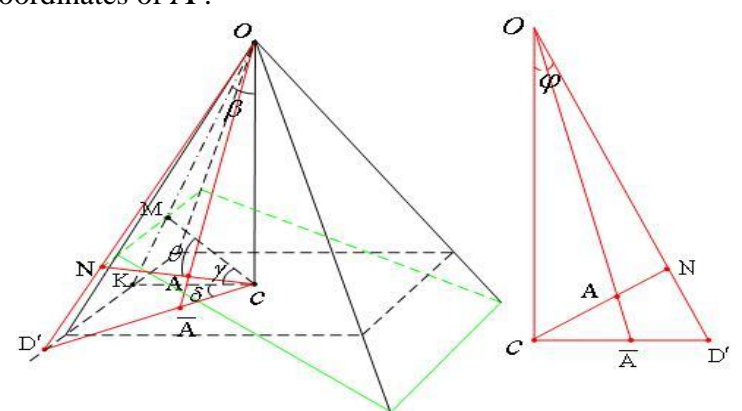

(a) Overall model (b) Abstract geometric relationship Figure4 Points in the upper plane

From the Figure4.b, the main difference starts from formulas (14). When the point is in the nether plane, $A C$ can be calculated by formula (19):

$$
A C=\frac{\overline{\mathrm{AC}} \cdot \mathrm{OD}^{\prime} \cdot \mathrm{NC}}{\mathrm{ON} \cdot\left(\mathrm{CD}^{\prime}-\overline{\mathrm{AC}}\right)+\overline{\mathrm{AC}} \cdot \mathrm{OD}^{\prime}}
$$

$\theta$ is the intersection corner of line $\mathrm{NC}$ and $\mathrm{MC}$ :

$$
\theta=\arccos \left(\frac{C M}{N C}\right)
$$

Then, the world coordinates of $A$ is:

$$
A\left(x_{1}, y_{1}\right)=(\mathrm{CA} \sin \theta, \mathrm{CA} \cos \theta)
$$

Through all above formulas, points position in world coordinates can be obtained, which gives a fundament base to modify the perception model of multimedia nodes.

\section{REAL FIELD OF VIEW}

A typical multimedia sensor node has directional perception model and the quality of surveillance service is usually affected by surrounding obstacles. If multimedia sensors cannot figure out their blind areas, the cooperative scheme will lose its usage. Therefore, in order to design efficient strategy for WMSNs, real perception model should be figured out firstly.

\subsection{Obstacle detection}

Before modifying the perception model, recognizing the images plays a key role for obstacle detection. This work is mainly to design processing flow and provide basic principles for detection by using sophisticated algorithms. Readers could select the proper image processing methods due to their various scenarios.

It is known that obstacles will cause occlusion in computer 
vision. To detect these objects, one underlying assumption is given that obstacles in image are more visually salient than the background [17]. Researchers tend to classify these observation behaviors as position saliency, area saliency, and intensity saliency [16]. As it is referred in above sectors, there are numerous multimedia sensors in WMSNs. The scenarios pictured are various and it becomes impossible to train these nodes learning the environment. Therefore, a general computational model is desired to incorporate different observation behaviors into one framework for obstacles detection.

There are numerous sophisticated algorithms for saliency detection $[16,17,20]$. In this part, the work is based on the achievements from J. Li [20], which adopted a saliency detection model by combining global information from frequency domain analysis and local information from spatial domain analysis. In the frequency domain, global information is used to model the nonsalient region. The repeating patterns, which are not distinctive in the scenarios, are suppressed by using spectrum smoothing. Then, in spatial domain, the algorithm enhances the regions, which are more informative by using center-surround mechanism similar to that found in the visual cortex. Finally, the outputs combine two channels to produce the saliency map and finish popping the potential objects.

After saliency detection is completed, characters of these popped areas are needed to be checked. In some cases, there is no need to regard all the salient regions as blind area. For example, when the emergent event requires alarm, sparse crown of a tree sometimes is acceptable in the perception model of this paper even though the algorithm may regard them as a salient object. Therefore, verification about whether the detected salient object should be regarded as the obstacles should be processed, since these obstacles will affect the surveillance task, through the following characters:

- The acreage of detected object ought to be large enough in image so that blind area cannot be ignored.

- The ratio of objects height and width should be limited in some range. If an object is just very long or high, then these objects may be not able to shelter our interesting events.

A monocular image will lose depth information in space (Figure5). Advanced knowledge about the obstacles' shape is unknown. It seems that there is no possibility to recover the 3D perception model from detected objects in 2D image. In order to deal with this issue and verify the detected object, a rectangle window approximating the irregular area is proposed. This procedure will assess whether the potential objects could affect the quality of surveillance service and be used in the next section as a preprocessing to cut down the difficulties of 3D recovery.

When rectangle approximation is assumed, the ratio of projection pixels in window is extremely important in terms of the obstacles characters stated before. If it is large enough, it is regarded these obstacles are capable to shelter our interested event and the blind area cannot be ignored. Otherwise, this occlusion cannot be considered as perceptional blind area.

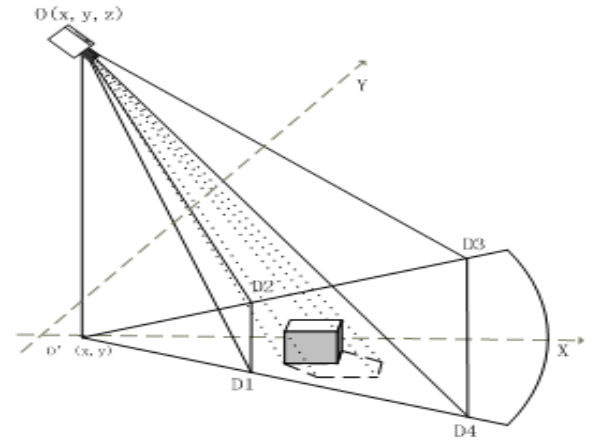

Figure5 Real perception model

Overall in this section, the main process in blind area seeking is divided into four steps:

Step1: Detect salient region to find potential occlusion area of a given image. In this step, there are many sophisticated methods which are not given elaborately in our paper. Readers who are interested in this could find more detailed method from papers $[16,17,20]$. In the selection of algorithm, the previous stated characters of objects are regarded as benchmark.

Step2: Segment the potential object region from backgrounds. Segmentation is to pop potential objects out from the background. After detecting salient region, the objects projection area could be segmented by binary image processing.

Step3: Rectangle approximation for segmented region. The projections of obstacles are irregular shape. Some reasonable simplifications are required so that $3 \mathrm{D}$ recovery from monocular image become realizable. This step is extremely important, which will be explained in following part.

Step4: Computation of efficient pixels percentage and ratio of height to width in approximation window. It is possible that the object occupies a large volume, but it will not affect our observation of events. That means if only caring about whether there are events occurring rather than the overall target, then some occlusions may be as well become acceptable.

This work is starting with obstacle detection firstly. However, it is hard to provide detailed methods in every step because the processed effect is based on real scenarios. Readers can just follow this processing flow and choose suitable algorithms.

\subsection{Maximum prism container}

As it is referred in above section, just from the irregular region in a monocular image, the real perception model can hardly be obtained. Rectangle window is used to approximate the region and solve this problem. With basic advanced knowledge of objects, this model can be modified (in Figure6).

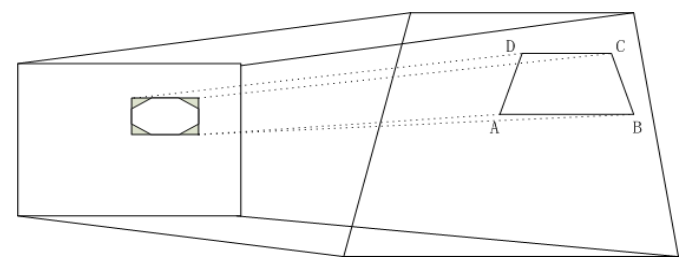

Figure6 The rectangle approximation 
In the Figure6, when using rectangle to approximate these shape, the rectangle window in image plane transforms to trapezium $\mathrm{ABCD}$ in the world coordinates.

The world coordinates of the points A $\left(x_{1}, y_{1}\right), \mathrm{B}\left(x_{2}, y_{2}\right)$, $\mathrm{C}\left(x_{3}, y_{1}\right), \mathrm{D}\left(x_{4}, y_{2}\right)$ can be computed by the formulas (18) or (21). It is easy to get the acreage of trapezium ABCD:

$$
\mathrm{S}_{1}=\frac{1}{4}\left(x_{1}-x_{2}+x_{3}-x_{4}\right) \cdot\left(y_{1}-y_{2}\right)
$$

As it is known, the obstacles are located on the ground. In the most cases, they are vertical to the ground plane, which means the bottom line of the projection is probably the intersecting line of earth and objects. Based on this knowledge, the approximate location of the objects can be calculated. Especially, rectangle window is utilized to approximate the region which makes locating procedure of interacting line become easier.

Numerous shapes will produce the same projection in the image. There is one situation drawing our attention. Looking at the Figure7.b, the bottom line of the projection may not be an intersecting line, which could be also caused by an edge of upper surface. It seems that this problem is hard to deal. For most of natural obstacles, the bottom surface is larger than topside of them. The situation in the Figure 7.b is very rare to see, because the center of gravity cannot be steady for natural objects. Therefore, in our perception model, another reasonable simplification and assumption is that the scenario of Figure7.b is very remote and the bottom line of the rectangle window is the interacting line. This will be the basement in our modification of the view field.

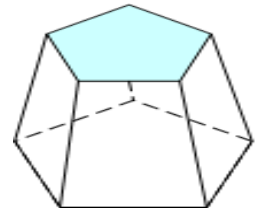

(a) Familiar obstacles

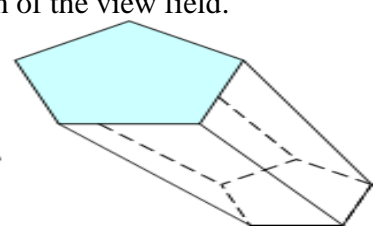

(b) Rare obstacles
Figure7 The feature of obstacles

From Figure8.b, it is shown that origin of projection line $\mathrm{M}^{\prime} \mathrm{N}^{\prime}$ is not unique. Various lines may also project into the same lines. How to find the perception blind area and make the calculated occlusion area contain the entire real occlusion field becomes a vital issue in our work.

To deal with this problem, maximum prism container $\mathrm{A}^{\prime} \mathrm{B}^{\prime} \mathrm{C}^{\prime} \mathrm{D}^{\prime}$ - $\mathrm{ABCD}$ is proposed in this paper (in Figure8).

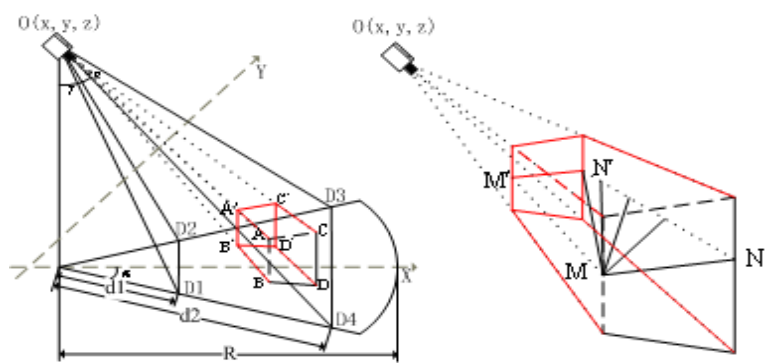

$\begin{array}{ll}\text { (a) Real sensing case (b) maximum prism container } & \text { (b) }\end{array}$ Figure8 Modification of perception model

If the size of the objects is out of this volume, the projection region is overrun by the rectangle window. So it is obvious that the maximum prism container contains all the possibilities of the obstacles. If height of the container is known, the upside acreage can be computed and the occlusion volume will be also obtained. In this step, our attention is drawn by height of this prism.
The projection procedure is shown in Figure8.a, which shows that when the $\mathrm{M}$ and $\mathrm{N}$ slides along the $\mathrm{OM}^{\prime}$ and $\mathrm{ON}^{\prime}$, the projection of them is the same.

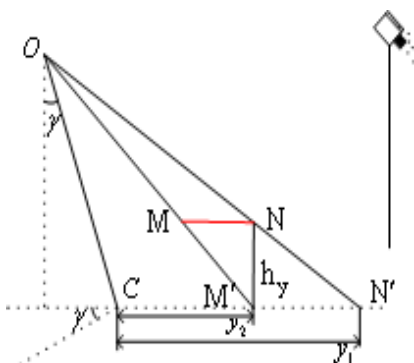

(a) 2D geometric relation

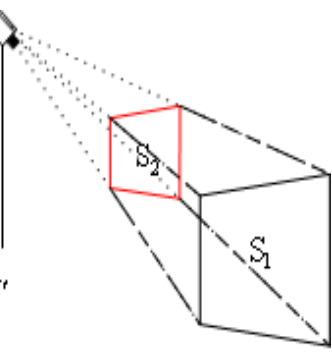

(b) occlusion areas
Figure9 The geometric relation of model

To find the height, the method is also inspired by the knowledge of obstacles in Figure7, which has been stated before: the obstacles cannot be as shown in Figure7.b and the bottom lines of the region are intersecting line. If all the natural objects are like what shown in Figure7.a, a reasonable and extreme case is that the obstacle is vertical to the ground. Then, the extreme height of prism container $\mathrm{h}_{\mathrm{y}}$ is shown in Figure9.a.

$$
\mathrm{h}_{\mathrm{y}}=\frac{y_{1}-y_{2}}{y_{1}+h \cdot \tan \gamma} \cdot h
$$

From this relationship model, the ratio of height in pyramid $\mathrm{O}-\mathrm{A}^{\prime} \mathrm{B}^{\prime} \mathrm{C}^{\prime} \mathrm{D}^{\prime}$ and in $\mathrm{O}-\mathrm{ABCD}$ can be computed:

$$
k=\frac{h-h_{\mathrm{y}}}{h}=\frac{y_{2}+h \cdot \tan \gamma}{y_{1}+h \cdot \tan \gamma}
$$

Therefore, it is easy to obtain the ratio of underside $A B C D$ acreage $S_{1}$ to topside $A^{\prime} B^{\prime} C^{\prime} D^{\prime}$ acreage $S_{2}$ :

$$
\frac{\mathrm{S}_{2}}{\mathrm{~S}_{1}}=k^{2}
$$

In terms of all above formulas, the occlusion volume is as shown in formula (26):

$$
\mathrm{V}_{\text {occlusion }}=\frac{1}{3} \mathrm{~S}_{1} \cdot h-\frac{1}{3} S_{2} \cdot k \cdot h=\frac{1}{3} h \cdot S_{1}\left(1-k^{3}\right)
$$

\section{PERFORMANCE EVALUATION}

In this section, laboratory experiments were designed to evaluate accuracy of the modified perception model in WMSNs.

\subsection{Experimental hardware}

Based on previous study, a LBT-based low-complexity image compression scheme is developed to overcome the computation and energy limitation of individual nodes [18]. The following research is to design a two-hop clustered image transmission scheme for maximizing the network lifetime [19]. In this network structure, there are two types of sensor nodes. One is ordinary sensor node which is used to measure scalar physical phenomena. Another one is advanced sensor node which occupied multimedia sensor. It is regarded as the cluster of sensor nodes, and this kind of sensor node is exactly what is studied in this paper. The structure of experimental node is shown in Figure10. 

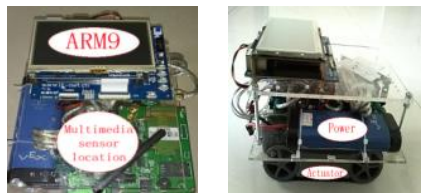

(a) Frontispiece (b) Right side

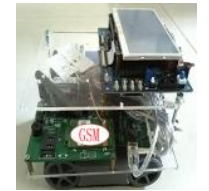

(d) Left side (d) camera

Figure10.a shows the frontispiece of sensor platform which contains five functional parts: sensors, processor, communication device, actuator and power unit. Figure10.b shows our multimedia sensor which will be located at sensors platform in Figure10.a. After optimizing the perception model, knowing the real sensing field, the collective and cooperative strategy will be designed. Then, the actuator executes corresponding assignment so as to enhance the quality of surveillance in WMSNs.

The main work in our paper is to modify the perception model of the multimedia sensor node, finding the blind area. This requires us to know the accurate parameters of our model. To achieve this, proposed model is applied to a more precise instrument, ignoring some irrelative hardware limitations temporarily. Therefore, multimedia sensor is located at the controlling system in Figure11, which could adjust the camera's attitude accurately.

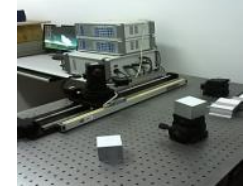

(a)System

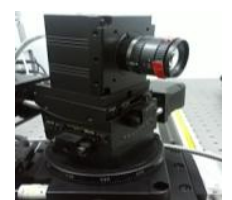

(b) Angle adaptor

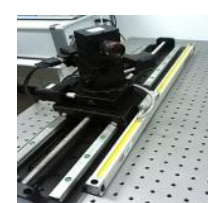

(c) Distance adaptor platform
In Figure11, this executor can be used to help precisely locate the camera. And this camera is installed in an adjustable platform. From Figure11.b, tilt angle $\gamma$ could range from $80^{\circ}$ to $90^{\circ}$. The location of camera in world coordinates $\mathrm{O}=(x, y, z)$ can be obtained in Figure11.c. All this adjustments are made through the software.

\subsection{Experimental results}

At the first stage of seeking the real sensing field, the obstacles detection shall be carried out in terms of our designed processing flow. In Figure12, the salient target is segmented using rectangle window to approximate this region, popping the objects accurately.

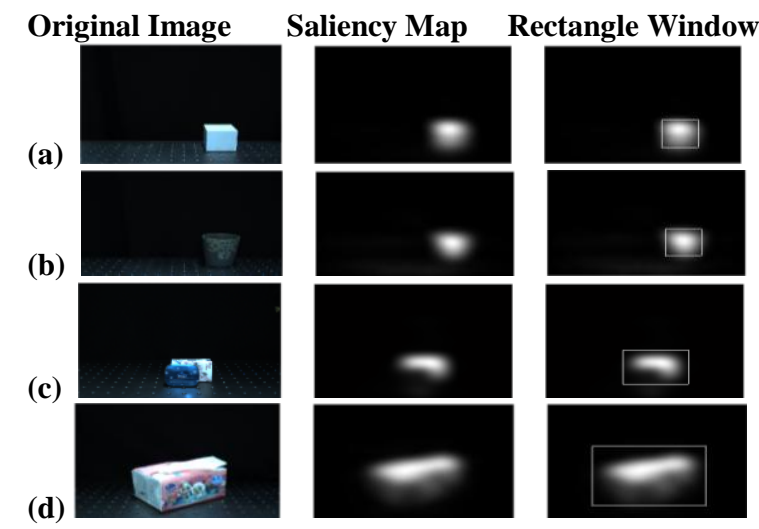

Figure12 Obstacle detection
In our experimental environment, point A shown in Figure7 is the most important point for our model because it decides the location of the obstacles in the image. As aim of this work is to figure out the occlusion area, whether distribution field of four points A, B, C and D includes the underside of object requires verification. In addition, the computational height of it ought to be higher than its real height. Therefore, the experimental work is divided into two parts. One is comparing the position error of point A while the other is to compute four points' location and the height to see whether the results contain all the blind area.

\section{A. The location of point $\mathrm{A}$}

In this part, a regular cube is used as obstacles because it can easily obtain the precise physical size so that comparison between computed results and perception model can be processed.

Firstly, adjusting the attitude of camera and making the tilt angle to be $80^{\circ}$. Affection of difference is what required. Therefore, maintaining Y coordinates of object but changing $\mathrm{X}$ coordinates with $5 \mathrm{~mm}$ every picture is performed. Due to the view scope of the camera, there are fifteen pictures with different object's locations in this experiment, and the location errors of XY are stated in Figure13.

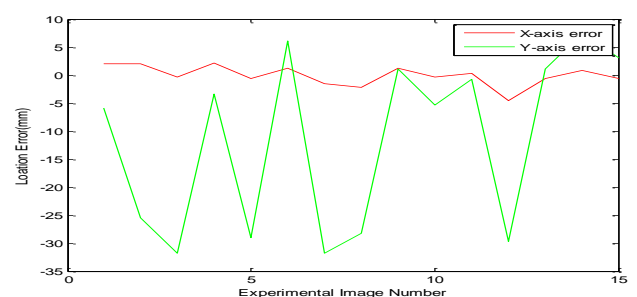

Figure13 Tilt angle $80^{\circ}$, changing location in $\mathrm{X}$-axis

In this experiment, it can be found that errors in $\mathrm{X}$-axis are limited in a small range. On the contrary, errors in Y-axis fluctuate dramatically.

Then, the second experiment is to fix $X$ coordinates but and make $\mathrm{Y}$ coordinates alter. The distance in $\mathrm{Y}$-axis is increasing by $5 \mathrm{~mm}$ every location. The results are shown in Figure 14

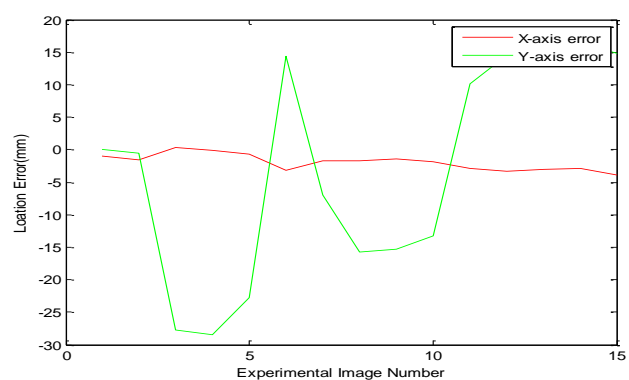

Figure14Tilt angle $80^{\circ}$, changing location in $\mathrm{Y}$-axis

Again, it can be obtained that errors in $\mathrm{X}$-axis are very small, while a large fluctuation of errors in $\mathrm{Y}$-axis can be seen in the Figure14.

In the following steps, to figure out the affection caused by tilt angle, $\gamma$ is adjusted to $75^{\circ}$ and same experiments are done. Results by changing the $\mathrm{X}$ coordinates are shown in Figure15. 


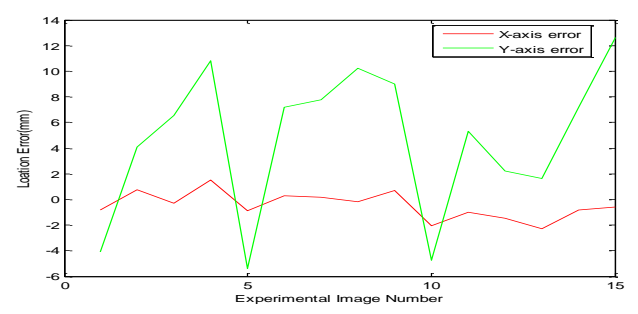

Figure15 Tilt angle $75^{\circ}$, changing location in $\mathrm{X}$-axis

Another experiment is carried out by fixing $\mathrm{X}$ coordinates but changing Y coordinates. The computational results are pictured in Figure16.

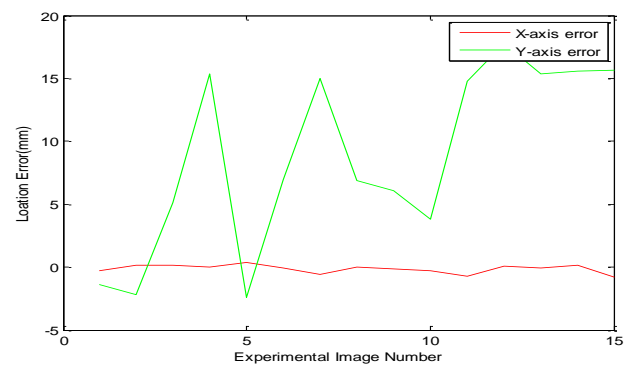

Figure16 Tilt angle $75^{\circ}$, changing location in $\mathrm{Y}$-axis

In an overall view of previous experiments, it shows that errors in $\mathrm{X}$ coordinate range in a limited extent. On the contrary, the error in Y coordinates fluctuates largely. It is noticeable that the location of the object has little effect on the result, while the degrees of tile angle will affect the fluctuation extent of the locating errors. Through analyzing the experimental phenomena, it can be observed there are some factors that play very important roles in location errors. Firstly, the computational errors are inevitable as they are generated from two factors: the accuracy of the saliency detection algorithm and the approximation rectangle window. If more precise results are to be obtained, a more advanced image processing algorithm is required which is not the emphasis in this paper. Secondly, the reason of errors fluctuation in Y-axis are much larger than they in $\mathrm{X}$-axis is that the computed $\theta$ in the experiments are quite small, this makes the value of $\cos \theta$ much larger than $\sin \theta$.Therefore, from formulas (18) and (21), it can be obtained this computed result will affect the location error in Y-axis fluctuating heavily, which could explain the experimental phenomena properly. Lastly, comparing the two groups with different tilt angles, fluctuation scope in Y-axis is increasing while the angle becomes larger. This is because larger tile angle will make the computed length of $C A$ be longer so that the fluctuation will become more dramatic.

\section{B. Occlusion area}

Another goal is to figure out the blind area so that effective collaborative strategy can be made in following research. Therefore, after points locating experiment, in this part world coordinates of the four points and the height of obstacles will be computed, which can employed evaluate the efficiency of the modified model.

The experimental samples are captured from our instrument in Figure11 and the pictures are shown in Figure12. Coordinates of the four points in approximation window are firstly computed. Then, in terms of our model, the height is computed. If it can be demonstrated that the enclosed area which formed by the four points contains the underside of the object and the computational height is higher than real height, it means that our modified model is effective and could help us figure out the real field of view.

The computational results are illustrated in Table1. As the shapes of objects' underside are irregular, some useful parameters are chosen. The real parameters of these in Table1 record the longest dimensions. In the meantime, the computed parameters are the length of the shortest side of the trapezium $A B C D$. If the computed volume shown in Table1 encloses the object, then it can be concluded that proposed method is efficient.

Table1 Inclusion relation

\begin{tabular}{|c|c|c|c|c|c|c|c|c|c|}
\hline \multirow[b]{2}{*}{ Pic. } & \multicolumn{2}{|c|}{ Real paremeters } & \multicolumn{6}{|c|}{ Computed parameters } & \multirow[b]{2}{*}{ Contair } \\
\hline & $\begin{array}{l}\text { Underside } \\
(\mathrm{mm} * \mathrm{~mm})\end{array}$ & $\begin{array}{l}\text { Height } \\
(\mathrm{mm})\end{array}$ & $\begin{array}{c}\mathrm{A} \\
(\mathrm{X}, \mathrm{Y})\end{array}$ & $\begin{array}{c}\mathrm{B} \\
(\mathrm{X}, \mathrm{Y})\end{array}$ & $\begin{array}{c}\mathrm{C} \\
(\mathrm{X}, \mathrm{Y})\end{array}$ & $\begin{array}{c}\mathrm{D} \\
(\mathrm{X}, \mathrm{Y})\end{array}$ & $\begin{array}{l}\text { Underside } \\
(\mathrm{mm} * \mathrm{~mm})\end{array}$ & $\begin{array}{l}\text { Height } \\
(\mathrm{mm})\end{array}$ & \\
\hline (a) & $65 * 65$ & 57 & $(20,492)$ & $(103,492)$ & $(164,810)$ & $(32,810)$ & $83 * 308$ & 86 & Yes \\
\hline (b) & $60 * 60$ & 87 & $(53,563)$ & $(144,563)$ & $(244,983)$ & $(90,983)$ & $91 * 420$ & 93 & Yes \\
\hline (c) & $72 * 20$ & 55 & $(-30,432)$ & $(81,432)$ & $(118,660)$ & $(-44,660)$ & $111 * 228$ & 75 & Yes \\
\hline (d) & $140 * 100$ & 70 & $(-83,444)$ & $(98,444)$ & $(207,1001)$ & $(-175,1001)$ & $181 * 565$ & 122 & Yes \\
\hline
\end{tabular}

Even though the computed area almost contains the blind area, it is still not very delighting to see that the computed errors are too large, especially the height of the underside. Noticeably, errors of underside not only contain the area which is occupied by the object's underside, but also enclose the invisible field which is caused by occlusion. Therefore, the computed field ought to be much larger than the object's underside. On the other hand, the error of underside is also decided by the saliency detection and the size of rectangle window. As said before, this image processing algorithm is not this work's emphasis, so researchers will try to optimize it in the future work. Moving to the height errors, it may be supposed this kind of errors is due to the model approximation error. Because real shape of obstacles is unknown, some approximation is required. That means this errors cannot be eliminated. As our aim is to ensure that the perception model could find the blind field, based on the reasonable assumption of obstacle in Figure7, a maximum redundancy of model is made, which shows that the computed height is higher than that in reality, so the model error is acceptable.

From the effect of experimental results, the modified model helps us figure out the real sensing region efficiently which could be a basement for the designing of the collaborative strategy.

\section{CONLUSION AND FUTURE WORK}

This paper proposed a real sensing model for multimedia sensors, which can be used as criteria to describe monitor service quality. This model utilizes rectangle to approximate segmented areas and considers hemline as the intersecting line between earth and objects. Using this intersecting line as benchmark, a maximum prism container is employed to find 
out blind areas. Experiments show that this algorithm can efficiently find out the real view field of nodes, even if it is a monocular image. Furthermore, this paper made contributions to $3 \mathrm{D}$ field of view study by considering physical obstacles in wireless multimedia sensors

\section{ACKNOWLEDGMENTS}

This work was supported by the National Science Foundation of PR China under Grant Nos. 61171136. We are grateful to all the reviewers for their insightful comments which improved the quality of this paper. Great appreciation is given to Fangqi $\mathrm{Wu}$, who provided excellent experimental equipment. Dr. Li offered beneficial suggestions for the section of image processing.

\section{REFERENCES}

[1] I. F. Akyildiz, T. Melodia, and K. R. Chowdhury, "A survey on wireless multimedia sensor networks," Comput. Netw. (Elsevier), vol. 51, no. 4, pp. 921-960, Mar. 2007.

[2] Tezcan, N., Wang, W. Self-Orienting Wireless Multimedia Sensor Networks for Maximizing Multimedia Coverage. In Proceedings of IEEE International Conference on Communications, ICC '08, Beijing, China, May 1923.pp. 2206-2210. 2008;

[3] C. Sha, R. Wang, H.Huang, and L.Sun, "An energy-saving strategy based on sleep scheduling and block transmission for wireless multimedia sensor networks," International Journal of Pervasive Computing and Communications, vol.6, no.2, pp. 1742-7371. 2010.

[4] Islam T. Almalkawi, M.G.Z.J., Wireless Multimedia Sensor Networks: Current trends and Future Directions. Sensors, doi: 10.3390/s100706662: pp. 6662 - 6717. 2010.

[5] R. Holman, J. Stanley, T.Ozkan-Haller, "Applying Video Sensor Networks to Nearshore Environment Monitoring," IEEE Pervasive Computing, vol 2, no. 4, pp. 14-21. 2003.

[6] Ma H D, Liu Y H. On coverage problems of directional sensor networks, Proceedings of the Int'l Conference on Mobile Ad-Hoc and Sensor Networks. LNCS 3794, Springer- Verlag, 721-731.2005.

[7] S. Soro, W. B. Heinzelman, On the coverage problem in video-based wireless sensor networks, in: Proc. of the Second Workshop on Broadband Advanced Sensor Networks (BaseNets'05), 2005

[8] R. Dai, I. F. Akyildiz, A Spatial Correlation Model for Visual Information in Wireless Multimedia Sensor Networks. IEEE TRANSACTIONS ON MULTIMEDIA, 11(6): pp. 1148-1159. 2009.

[9] Ammari H M, Das S K. Critical Density for Coverage and Connectivity in Three-Dimensional Wireless Sensor Networks Using Continuum Percolation[J], IEEE
Transactions On Parallel And Distributed Systems, 20, (6): 872-885, 2009

[10] Ma H D, Zhang X, Ming A N. A Coverage-Enhancing Method for 3D Directional Sensor Networks, Proceedings 28th IEEE Conference on Computer Communications (IEEE INFOCOM 2009), Rio de Janerro, Brazil, 19-25.pp: 2791-2795 April 2009.

[11] Kang C W, Chen J H. Multi-objective evolutionary optimization of 3D differentiated sensor network deployment. GECCO (Companion) pp: 2059-2064.2009

[12] XIAO Fu, WANG Ru-chuan, SUN Li-juan, WU Shuai. Research on the three-dimensional perception model and coverage-enhancing algorithm for wireless multimedia sensor networks. The Journal of China Universities of Posts and Telecommunications, DOI: 10.1016/S10058885 (09) 60580- X: pp. 67-72. 2010.

[13]] D. A. Forsyth and J. Ponce, Computer Vision: A Modern Approach. Englewood Cliffs, NJ: Prentice-Hall, 2002.

[14] N. Patwari, J.N. Ash, S. Kyperountas, A.O. Hero Iii, R.L. Moses, N.S. Correal, Locating the nodes: cooperative localization in wireless sensor networks, IEEE Signal Processing Magazine 22 (4) pp: 54-69. 2005

[15] L. Mihaylova, D. Angelova, D.R. Bull, C.N. Canagarajah, Localisation of Mobile Nodes in Wireless Sensor Networks with Time Correlated Measurement Noises, in IEEE Transactions on Mobile Computing. pp: 44-53. 2011.

[16] Wei Zhang, Q. M. Jonathan Wu, Guanghui Wang, Haibing Yin, An Adaptive Computational Model for Salient Object Detection, IEEE TRANSACTIONS ON MULTIMEDIA,12(4):300-316, JUNE 2010

[17] HUANG TieJun, TIAN YongHong, LI Jia, YU HaoNan, Salient region detection and segmentation for general object recognition and image understanding, Information Sciences, doi: 10.1007/s11432-011-4487-1, Vol. 54 No. 12: 2461-2470, December,2011

[18] Q. Lu, W. Luo, J. Wang, B. Chen, "Low-complexity and energy efficient image compression scheme for wireless sensor networks", Computer Networks (Elsevier) 52 (13) (2008) 2594-2603.

[19] Z. Zuo, Q. Lu, W. Luo, "A two-hop clustered image transmission scheme for maximizing network lifetime in wireless multimedia sensor networks", Computer Communication (Elsevier) doi:10.1016/j.comcom. 2011.07.009.

[20] J. Li, M. D. Levine, X. An , X. Xu and H. He, Visual Saliency Based on Scale-Space Analysis in the Frequency Domain. JOURNAL OF LATEX CLASS FILES, VOL. 6, NO. 1, JANUARY 2007. 